http://dx.doi.org/10.4995/ANIAV.2015.560

Torrecilla Patiño, Elia.

Investigadora, Universidad Politécnica de Valencia, Departamento de Escultura, Laboratorio de Creaciones Intermedia.

\title{
La geolocalización de la deriva urbana como forma de lectura de espacios híbridos.
}

\author{
TIPO DE TRABAJO \\ Comunicación.
}

PALABRAS CLAVE

Deriva, ciudad, espacios híbridos, arte, matemáticas, GPS .

KEY WORDS

Drift, city, hybrid spaces, art, math, GPS.

\section{RESUMEN}

A partir de un trabajo práctico realizado con motivo de la participación en el proyecto Modelización matemática de formas escultóricas complejas. Investigación interdisciplinar experimental de arte contemporáneo y matemáticas, que se encuentra dentro del Programa de Apoyo a la Investigación y Desarrollo de la U.P.V (PAID-05-12), se plantea introducir el concepto de espacio híbrido, concretamente el espacio urbano y sus dos dimensiones: la física y la virtual.

La propuesta llevada a cabo, junto con la colaboración de varios miembros del Instituto Universitario de Matemática Pura y Aplicada de la UPV, busca establecer una interacción entre el campo del arte y las matemáticas aplicando las derivadas de una función en un punto dado a una serie de derivas (desplazamiento sin rumbo): tras la realización de varias caminatas geolocalizadas mediante una aplicación móvil, se procede a la recopilación de datos de posicionamiento espacial, temporal y de emociones que afectan al rumbo de la propia deriva. Una vez escogida la emoción (una serie de textos encontrados) se realiza una búsqueda de los datos de espacio y tiempo de cada instante para posteriormente derivarlo y revelarnos de ese modo, la velocidad media alcanzada en el momento concreto de la emoción correspondiente a cada deriva, un acontecimiento que aporta una dimensión humana a una representación abstracta.

Con la introducción de este proyecto, deambularemos por un espacio que fluctúa entre lo físico y lo virtual, donde el primero se transforma en una red de coordenadas que se sitúa entre el espacio vivido y el espacio abstracto; una experiencia en un espacio híbrido o en una ciudad-interfaz a través de un cuerpo-software.

\section{ABSTRACT}

From a practical work done in connection with the participation in the project tittled Mathematical modeling of complex sculptural forms. Experimental interdisciplinary research of contemporary art and mathematics, which is within the Program to Support Research and Development UPV (PAID-05-12), we propose to introduce the concept of hybrid space, namely the urban space and its two dimensions: the physical and the virtual.

The proposal carried out, with the collaboration of several members of the Institute of Pure and Applied Mathematics, seeks to establish an interaction between the field of art and mathematics applying derivative of a function at a given point a series of drifts (moving aimlessly): after performing several geo walks through a mobile application, proceed to the 
collection of spatial positioning data, temporal and emotions that affect the direction of the drift itself. After selecting the emotion (a series of founded texts) a search of the data in space and time of each moment later I refer you and reveal thereby performed, the average speed achieved at the particular time of the corresponding emotion to each drift, an event that brings a human dimension to an abstract representation.

With the introduction of this project will drift by a space ranging between the physical and the virtual, where the first transformed into a network of coordinates that is between lived space and abstract space; experience in a hybrid space or in a city-interface through a body-software.

\section{CONTENIDO}

\section{INTRODUCCIÓN}

Desde el siglo XIX hasta la actualidad, la ciudad ha vivido una serie de transformaciones, evolucionando y dando lugar a su comprensión difusa, pues se encuentra representada a partir de fragmentos, un hecho que se vuelve evidente cuando analizamos su presencia en el espacio virtual, cuyo aspecto está conformado a partir de fracciones fotográficas en las cuales se elimina cualquier indicio espacial o temporal. Ante este hecho, el artista emplea tanto el entorno urbano como el virtual como medio y soporte para sus creaciones, encontrando en la actividad del caminar un medio que le permite analizar la realidad circundante, pues el paseo se convierte en un método de contacto y observación directa con lo que le rodea. Pero nuestra forma de percibir el mundo que habitamos se ha visto profundamente alterada con la aparición de dos innovaciones tecnológicas: Internet y los dispositivos móviles.

Aproximadamente dos décadas después de la propagación del uso del primero y tras 15 años desde la incursión a nivel masivo de los segundos, han sido numerosos los análisis que se han realizado sobre la nueva realidad que habitamos. Los datos y las imágenes aumentan y reconfiguran nuestra experiencia del entorno dando lugar a una especie de paisaje móvil y hoy, más que nunca, el paisaje es una combinación próxima a la interfaz, como si se tratara de una pantalla más de las muchas que hoy rodean al individuo.

Gracias a la evolución del ciberespacio y a los numerosos estudios que se han llevado a cabo para intentar analizar y comprender esta nueva realidad, se ha podido observar cómo el espacio urbano ha sido configurado en este espacio virtual, dejando evidencia de la fragmentación que presenta la ciudad postmoderna. Varias aplicaciones son las que facilitan la comprensión del espacio urbano en el ciberespacio, siendo éstos los denominados medios locativos, que son aquellos que combinan tecnologías y servicios basados en la localización. A través de dispositivos como el GPS (Global Position System), se ofrece información sobre un espacio físico, y debido a su evolución y popularización, están aportando nuevos significados y conceptos relacionados con el espacio.

En este artículo se comentará un proyecto que se inscribe dentro del desarrollo de la Tesis Doctoral que lleva por título Redefinición de la práctica artística del paseo en el espacio urbano a partir de la influencia de los medios locativos, que se encuentra en proceso de realización y que se está llevando a cabo gracias al Programa de ayudas para la formación de personal investigador (FPI) de la UPV, y que surge, concretamente gracias a la colaboración entre el Departamento de Matemática Aplicada de la UPV y el Grupo de Investigación del Laboratorio de Creaciones Intermedia, dentro del Departamento de Escultura de la Facultad de Bellas Artes de la UPV, con el que se trata de explorar el amplio territorio que existe entre el arte y las matemáticas a través del proyecto Modelización matemática de formas escultóricas complejas. Investigación interdisciplinar experimental de arte contemporáneo y matemáticas.

\section{DESARROLLO}

El trabajo que aquí presento lleva por título Deriva(da)s, que es un juego de palabras formado por la unión entre deriva y derivada, es decir, entre la unión de una acción artística y una operación matemática.

Para comenzar, vamos a dar una breve descripción de ambos conceptos.

DERIVA: En 1958, uno de los fundadores de la Internacional Situacionista, Guy Debord, escribió La Teoría de la Deriva, a la que definió como "una técnica de paso ininterrumpido a través de ambientes diversos" 1 , explicando que la o las personas entregadas a la deriva, renuncian a sus motivaciones personales de desplazamiento habitual para dejarse llevar por las solicitaciones del terreno y por los encuentros que a él corresponden. El concepto de deriva lleva implícito el reconocimiento de efectos de naturaleza psicogeográfica, porque permite realizar una lectura global de las ciudades industriales, y la afirmación de un comportamiento lúdico-constructivo que la opone en todos los aspectos a las nociones clásicas de viaje y de paseo. 
En la Teoría de la Deriva, Debord comenta algunos aspectos a tener en cuenta, como por ejemplo la importancia que juega el azar durante el desplazamiento, porque a medida que el sujeto que deriva va siendo consciente de la distribución psicogeográfica del lugar por el que deambula, el azar va perdiendo efecto en el proceso de selección de recorridos y creación de situaciones en la deriva, una acción que se va articulando en función del deseo y la voluntad del sujeto.

En cuanto al número de participantes, explica que se puede derivar en solitario o en grupos pequeños de dos o tres personas que compartan un mismo estado de conciencia. En cuanto a la duración de una deriva, aclara que no es demasiado importante, pues es común distraerse una o dos horas para dedicarlas a ocupaciones banales. A menudo, la deriva se desarrolla en determinadas horas deliberadamente fijadas, así como en breves instantes fortuitos o durante varios días sin interrupción. Y respecto al clima, aunque las condiciones climáticas influyen sobre la deriva, no son determinantes más que en el caso de lluvias prolongadas que la impiden casi por completo, pero las tempestades y demás precipitaciones resultan más bien propicias. En cuanto al espacio, será más o menos vago o preciso dependiendo de si se busca el estudio del territorio o la vivencia de emociones desconcertantes. La extensión máxima del espacio de la deriva no excede el conjunto de una gran ciudad y sus afueras y su extensión mínima puede reducirse a una unidad pequeña de ambiente: barrio, manzana...

\section{DERIVADA:}

En el campo de las matemáticas, la derivada de una función en un punto dado, es una medida de la rapidez con la que cambia el valor de dicha función matemática, según cambie el valor de su variable independiente y se calcula como el límite de la rapidez de cambio media de la función en un cierto intervalo, cuando el intervalo considerado para la variable independiente se vuelve cada vez más pequeño. Suele emplearse para estudiar el movimiento: si una función representa la posición de un objeto con respecto al tiempo, su derivada es la velocidad de dicho objeto.

\section{EL PROCESO:}

En el proyecto Deriva(da)s, se emplea la técnica de la deriva, un procedimiento que fue diseñado como método de lectura de la ciudad, donde las interrupciones del azar son constantes.

Durante la deriva, el sujeto que deambula va tomando conciencia del espacio y su psicogeografía, definida por Debord como "la teoría de uso combinado de las artes y de la técnica para la construcción integral de un ambiente en relación dinámica con experimentos en el comportamiento", proponiendo la búsqueda de emociones desconcertantes mediante la desorientación, porque cuando esta se busca, las interferencias que se producen son múltiples2.

De este modo, el método escogido para la realización de las cinco derivas que componen este trabajo son las siguientes:

1. Todas las derivas se han visto afectadas por el azar. La elección de los caminos recorridos se llevó a cabo en función de diferentes situaciones como los flujos de gente, pasos cortados por el tráfico, aspectos estéticos...

2. Las derivas fueron realizadas de forma individual.

3. Cada caminata tiene una duración diferente, oscilando todas ellas entre las 2 y las 6 horas, transcurso determinado por la capacidad física y mental.

4. El clima no se ha tenido en cuenta pero la última deriva realizada (Deriva Al Agua) se vio inevitablemente afectada por la lluvia, lo que condicionó toda la jornada de desplazamiento.

5. Todas las derivas se realizaron en diferentes espacios de la ciudad de Valencia, tanto en el centro como en la periferia.

Cada uno de los cinco días, se partió de un lugar diferente para ir "a la deriva", sin objetivo alguno de llegada, siendo cada una de ellas geolocalizada de principio a fin a través de la aplicación móvil EveryTrail3, que, a través del GPS, que se basa en la determinación de la distancia entre un punto (el receptor) a otros de referencia (los satélites), convierte la señal en datos de posición, velocidad y tiempo. 


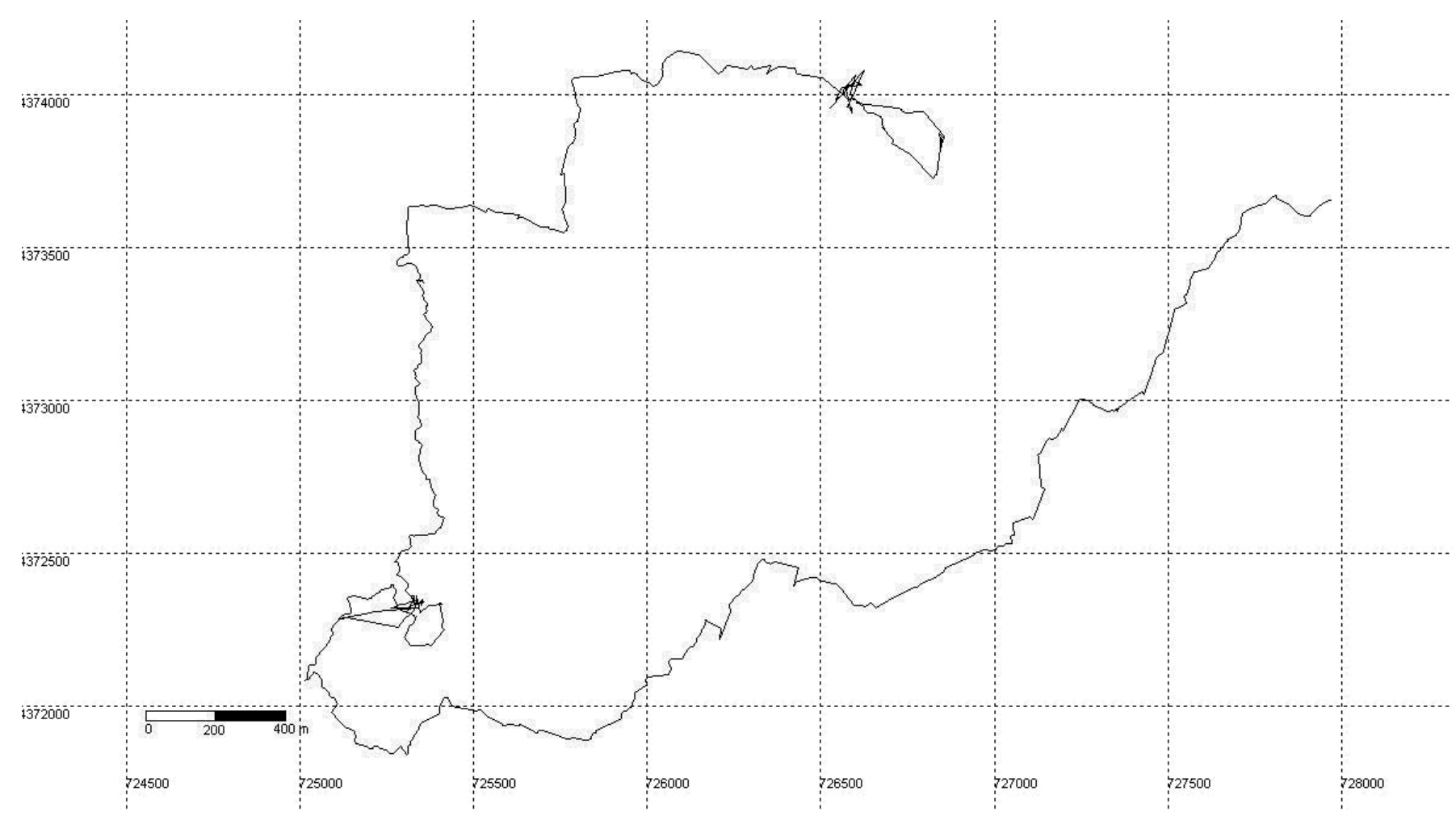

Imagen I: Captura del recorrido de una deriva

Posteriormente, con el programa informático GPS TrackMaker4 se tradujo el recorrido en datos de posicionamiento:

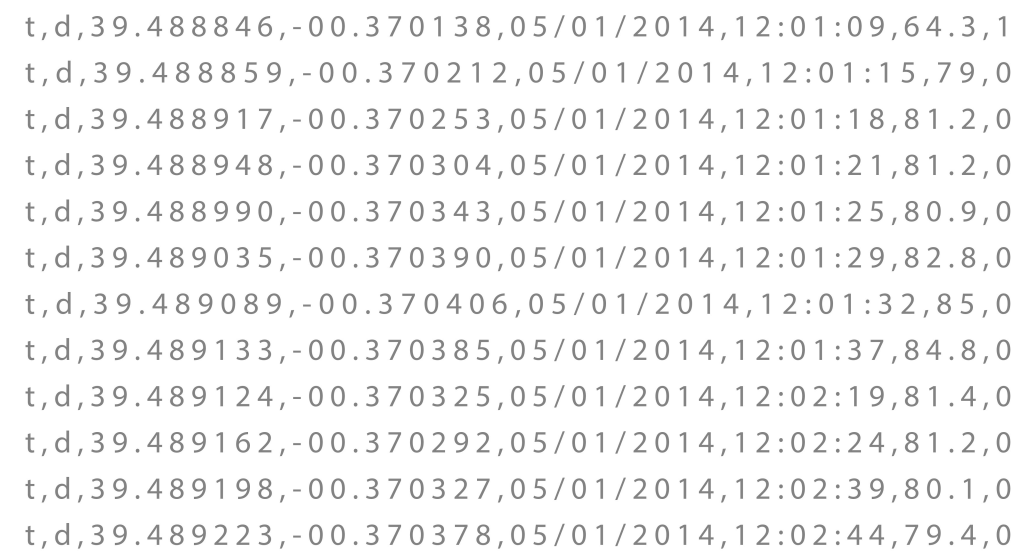

Imagen II: Fragmento de la recopilación de datos de localización recogidos de una deriva.

Como se comentaba anteriormente, la deriva busca establecer contacto con las emociones del lugar y es una metodología para formular mapas psicogeográficos, por ello, cada una de las derivas realizadas para este proyecto se han guiado por acontecimientos que han cambiado el rumbo del desplazamiento. Para la realización de la operación matemática de la derivada, que consiste en calcularel movimiento en un punto dado, se ha escogido una emoción, que en este caso son una serie de textos encontrados en las calles y que de alguna manera respondían a ciertas cuestiones planteadas en durante el discurso de la propia deriva. 


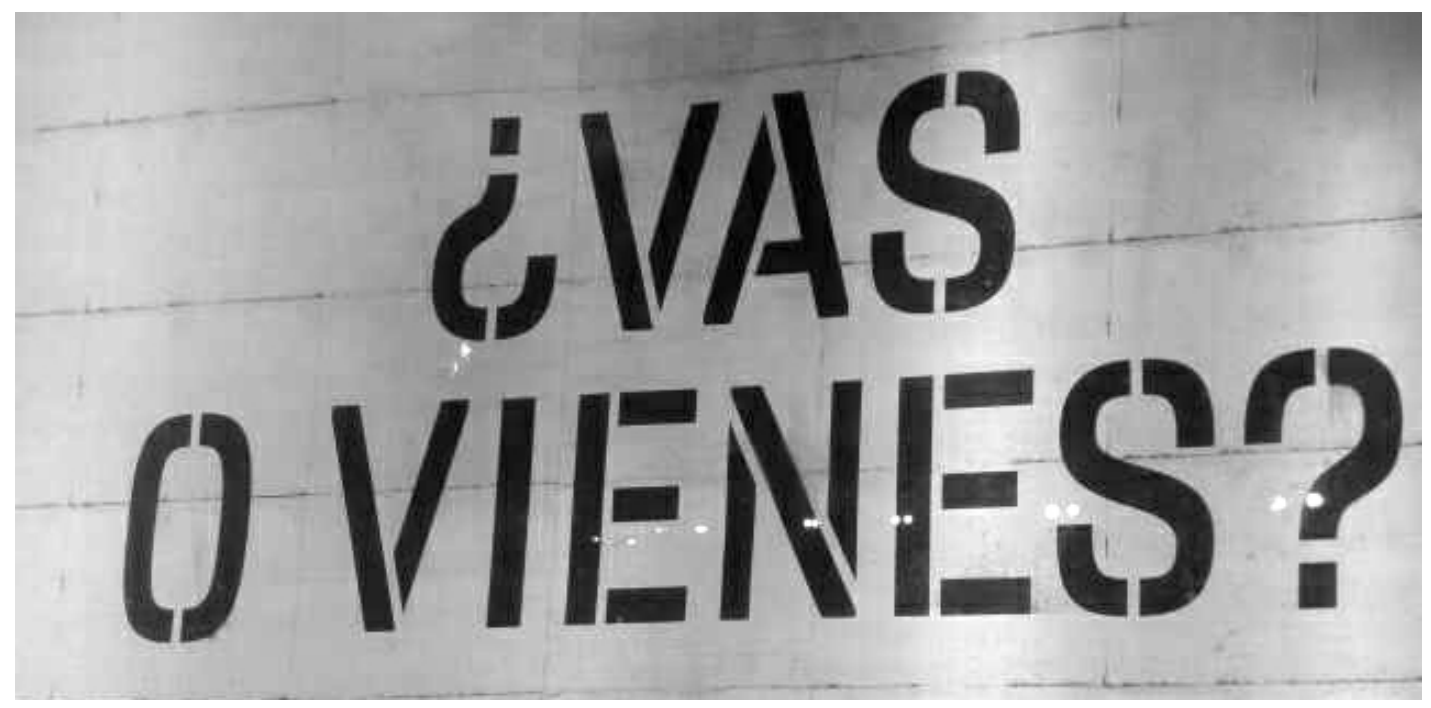

Imagen III: Emoción escogida de la primera deriva presentada.

Puesto que cada uno de los instantes o emociones escogidos estaban situados en un punto concreto del espacio y fueron encontrados en un tiempo preciso, todos ellos tienen una posición espacio-temporal recogida a través de la aplicación móvil. Así,los datos que corresponden a la emoción “¿VAS O VIENES?” son: t,d,39.482288,-00.375016,05/13/2014,21:45:46,100,0

Con todos los datos de posición recopilados de cada deriva, los colaboradores miembros del Instituto Universitario de Matemática Pura y Aplicada, a través de una aplicación informática, realizaron un cálculo para obtener el vector de una posición dada (la posición en el momento que la emoción tuvo lugar).

Los datos obtenidos de esta deriva en concreto fueron:

Posición: (39.4823, -0.375016) Hora (pasada a sistema decimal): 21.7628 Vector derivada: $(0.0314253,-0.0164625)$

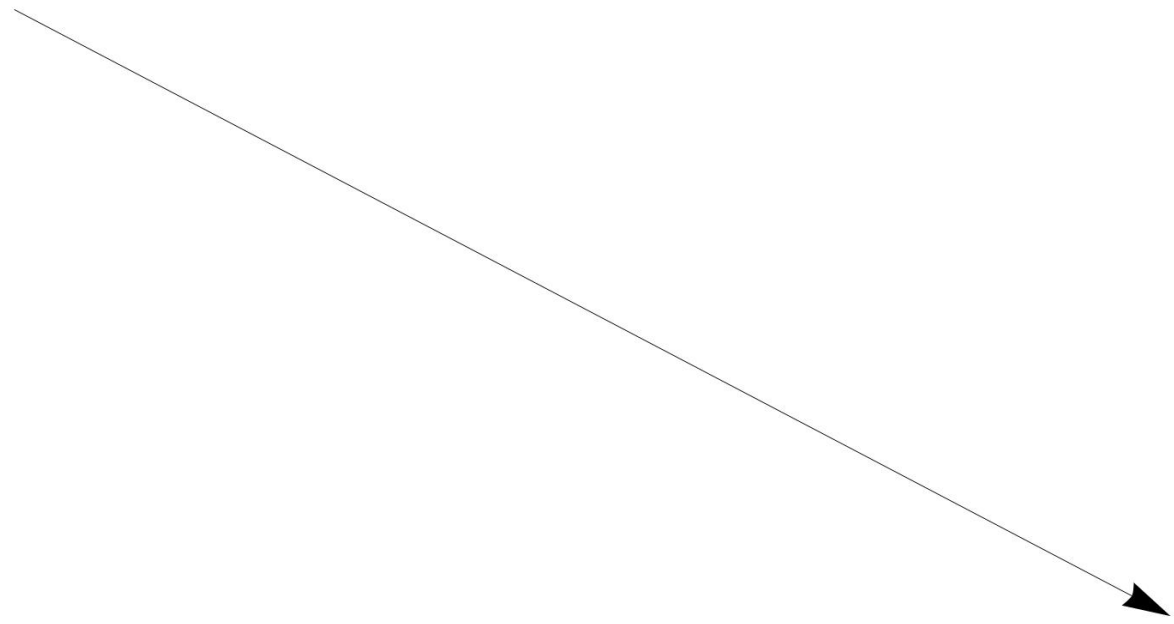

Imagen IV: Representación gráfica de la derivada

Una vez calculada la operación de la derivada, se procedió a la impresión del conjunto de datos de geolocalización. Puesto que cada dato es la posición de un punto en el espacio, se decidió imprimir todas esas líneas de texto con una impresora matricial, que estampa mediante un sistema de puntos y cuyo proceso fue documentado en vídeo. 


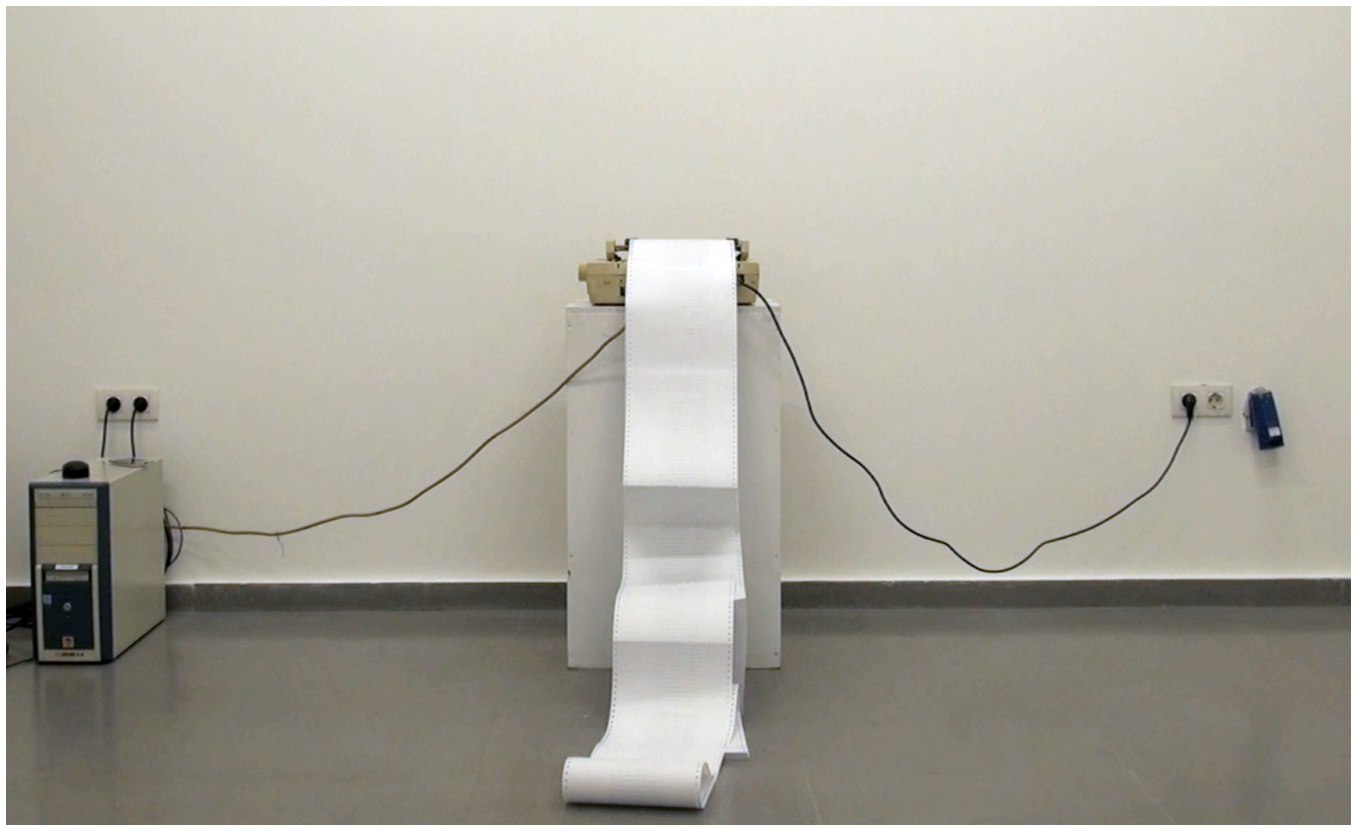

Imagen V: Captura del vídeo que documenta la impresión de datos

\section{CONCLUSIONES}

La Teoría de la Deriva, propuesta por los situacionistas a finales de la década de los 50, surge como reacción ante el aburrimiento en la ciudad, haciendo un llamamiento al "dejarse llevar" y, rechazando la representación formal de la urbe que muestran los planos, trazaban recorridos psicológicos según las diversas experiencias urbanas, prestando atención a los detalles, a la lectura lúdica del espacio, al disfrute y a la transformación de la ciudad, dejándose hechizar por la sorpresa y el deseo, y convirtiéndose en una práctica fundamental para entrar en contacto con el espacio circundante.

Cada vez resulta más difícil disfrutar de la ciudad; las calles se han convertido en espacios de tránsito por los que vagan multitud de sujetos que son movidos, en su mayoría, por impulsos consumistas y constantemente conectados a través de sus dispositivos móviles a través de los cuales se produce un acto de comunicación, de relaciones sociales y de intercambio de información que en la mayoría de los casos aísla al usuario del espacio que le rodea provocando un cambio en la experiencia que se tiene del lugar. Por otra parte, es frecuente el empleo de aplicaciones de geolocalización para desplazarnos de un punto a otro guiados por las directrices que dictan estas herramientas, lo que dificulta la capacidad de desorientación y de perderse, imposibilitando el efecto sorpresa y la creación de situaciones.

A través de esta experiencia práctica se han realizado una serie de recorridos que se sitúan entre el espacio físico y digital, por la combinación del uso de la ciudad y la aplicación móvil basada en la geolocalización empleada, que ofrece información digital sobre un espacio físico. Esto da lugar a lo que André Lemos denomina territorios informativos5, término que emplea para referirse a aquellas áreas en las que el flujo de información en la intersección entre el espacio virtual y el espacio urbano se controla digitalmente, produciendo de este modo una heterotopía6. Por territorio informativo, Lemos entiende el área de control del flujo de información digital en su intersección con un área física, adquiriendo de este modo una nueva capa de información digital, formando un nuevo territorio creado por redes electrónicas y dispositivos móviles.

Esta interacción entre ambos espacios (físico y digital) da lugar a los llamados espacios híbridos, que ofrecen nuevas experiencias de las dimensiones espacial y temporal. Cuando hoy hablamos de espacio público, podemos decir que hablamos de espacio híbrido, puesto que en él la comunicación se realiza empleando tanto interfaces físicas como digitales. Diego Díaz, en su Tesis Doctoral De la plaza al chat7 habla del espacio público como interfaz de comunicación.

Díaz explica que desde el punto de vista de la tecnología informática, la interfaz se define como como una zona de contacto y conexión entre dos componentes físicos, entre dos aplicaciones o entre un usuario y una aplicación, por ello, si entendemos que la interfaz es el lugar en donde se produce la interacción y los intercambios, podemos hablar del espacio público como una interfaz de comunicación. 
En la actualidad nos desplazamos por el espacio urbano acompañados por dispositivos tecnológicos móviles a través de los cuales nos comunicamos con otros o mediante los cuales nos orientamos, relacionándonos con ellos incluso como una extensión de nuestro propio cuerpo. Para McLuhan8, la tecnología es la creación de un medio del que no disponemos cuando nacemos; así, la radio es una extensión del oído, la TV una extensión de la vista... y ello, "rompe los equilibrios naturales para restituir al individuo en la totalidad de sus sensaciones". La ciudad se nos presenta como un espectáculo del que no somos solamente observadores, sino que formamos parte de él y compartimos escenario con los demás participantes. Muy a menudo, nuestra percepción de la ciudad no es continua, sino más bien parcial, fragmentaria, mezclada con otras preocupaciones. Casi todos los sentidos están en acción y la imagen es la combinación de todos ellos9, una imagen que nos ofrece diferentes lecturas, un texto dispuesto para ser descifrado por los habitantes. Este empleo de la metáfora del texto para examinar el espacio, ha sido utilizado por los geógrafos y demás investigadores sociales en la interpretación de los diferentes procesos socio-espaciales.

El conjunto de signos que nos ofrece una ciudad, forma un texto, y un texto se construye en un espacio (la página en blanco). El historiador y filósofo francés Michel de Certeau10 nos explica que la página en blanco es un lugar de producción para el sujeto, un espacio aislado y alejado de su actividad cotidiana. Se trata de una superficie autónoma situada bajo su ojo, y como si de un urbanista se tratara el sujeto tiene que manejar el espacio, propio y distinto, donde poner en obra su voluntad. El texto se construye en la página en blanco a partir de fragmentos y materiales lingüísticos dispuestos de una manera mediante la cual se produce un orden. El lector es activo, productor; es un cazador furtivo de sentidos, de re-interpretaciones, y si entendemos la ciudad como un conjunto de signos que forman un texto, podemos entender el acto de caminar como una forma de lectura y de escritura al mismo tiempo, pues a cada paso vamos dejando signos que contribuyen a la creación de una ciudad-palimpsesto. Caminar es un proceso de apropiación del espacio por parte del peatón que deviene lector, y también escritor. En esta propuesta se manifiesta la escritura digital que se produce con cada movimiento que realizamos. Somos puntos en el espacio controlados por las tecnologías que proporcionan los datos de nuestro posicionamiento, dejando constancia de las coordenada espaciales, temporales y de velocidad, registrando en el espacio virtual los desplazamientos físicos. Por ello, como se comentaba al principio de este artículo, el proyecto Deriva(da)s ha permitido realizar una deambulación a través del arte y las matemáticas, por un espacio que fluctúa entre lo físico y lo virtual, donde el primero se convierte en una red de coordenadas que se sitúa entre el espacio vivido y el espacio abstracto, dando lugar una experiencia en un espacio híbrido o en una ciudad-interfaz a través de un cuerpo-software.

\section{FUENTES REFERENCIALES.}

DEBORD, Guy: Teoría de la Deriva, 1958. Texto aparecido en el \#2 de Internationale Situationniste. Traducción extraída de Internacional situacionista, vol. I: La realización del arte, Madrid: Literatura Gris, 1999.

[http://www.ugr.es/ silvia/documentos\%20colgados/IDEA/teoria\%20de\%20la\%20deriva.pdf ]. [Fecha de consulta: 18/03/2015]

DIAZ GARCÍA, Diego José: De la plaza al chat: Análisis de las transformaciones del espacio público desde la práctica artística neomedial. Universidad Politécnica de Valencia, Facultad de Bellas Artes de San Carlos, Departamento de Escultura, Valencia, 2007.

LEMOS, André: Medios Locativos y Territorios Informativos. Comunicación Móvil y Nuevo Sentido de los Lugares. Una Crítica sobre la Espacialización en la Cibercultura, 2008.

[https://www.google.it/url?sa=t\&rct=j\&q=\&esrc=s\&source=web\&cd=1\&cad=rja\&uact=8\&ved=0CCEQFjAA\&url=http\%3A\%2F\%2Fmedialabprado.es\%2Fmmedia\%2F8844\&ei=FYlyVZvIB8XWOqiTgIAI\&usg=AFQjCNEZxKwWfcfRzpcCYArz3E6cOeQzvQ\&sig2=KfbAvH7K8ESp_RV1NDiFA\&bvm=bv.91071109,d.bGg]. [Fecha consulta: 29.02.2015]

LÓPEZ RODRÍGUEZ, Silvia: Orientación y desorientación en la ciudad. La teoría de la deriva. Indagación en las metodologías de evaluación de la ciudad desde un enfoque estético-artístico, 2005 [http://digibug.ugr.es/handle/10481/823\#.VTKAwVyWG2w]. [Fecha consulta: 03.04.2015]

LYNCH, Kevin: La imagen de la ciudad. Editorial Gustavo Gili SL, Barcelona, 1984, 1998.

MCLUHAN, Marshall: Understanding Media: The Extensions of Man. Gingko Press, California, 1964. 